\title{
Interactive comment on "Ten years of atmospheric methane from ground-based NDACC FTIR observations" by Whitney Bader et al.
}

\section{Anonymous Referee \#1}

Received and published: 20 September 2016

Review of "Ten years of atmospheric methane from ground-based NDACC FTIR observations" by Bader et al.

This paper determines trends in total column methane from a subset of globally disperse NDACC FTIR sites over a relatively short time period 2005-2014. The data are compared to the GEOS-Chem CTM for trends and via tagged emissions an effort is made to determine year over year changes in methane sources that may contribute to the observed columns.

General / Major Comments

The paper is concise restricting itself to 10 stations and 10 years of data but leaves a considerable amount of similar data out. There are more stations with archived data and many stations have data dating back to the mid 1990's and earlier. A description 
of this larger dataset would be considerably more illuminating and reflective of the longevity and efforts of the NDACC network. In a similar context of under reporting, Fig 3 proves stratospheric information is contained in these data yet these trends are not explored. Relative trends in the troposphere vs the stratosphere would be unique and important. Its not clear what advantage Fig 3 delivers when the information is ignored. Instead ACE-FTS date is invoked to discuss any stratospheric trend. While this data record is also long, it is sparse and not as long as the NDACC record. Why was the CTM results stopped at 2013? This appears arbitrary and again leaves out information

Overall the manuscript is very well written. It leaves much analysis from this rich dataset underutilized and/or undeveloped.

There is too little discussion of total uncertainty in the $\mathrm{CH} 4$ columns. References to two other papers does not seem adequate in general and in particular given the use of noise in Wollongong data trends later in the paper as a reason for a mismatch in trend (Pg 9). Were errors even used in the trend analysis? If so were then nominal values or real i.e. derived from calculations for each measurement?

$\mathrm{Pg} 6$ Is a discussion of a type of normalization process with a stated purpose "to characterize the possible impact of the choice of the microwindows and spectroscopy on the retrieved methane". A set of data from JFJ is fit by all other stations using their local method and hence a relative station bias to Jungfraujoch is determined and consequently applied to each respective dataset. 1. This does not "characterize" effects of retrieval parameters. There is no further discussion to investigate this issue i.e. there is no actual characterization. There may be valid reasons to use differing parameters at different sites (e.g. interfering species) that may have unintended consequences when fitting JFJ spectra. A characterization exercise might reveal this. 2. The station bias values are not given, only a mean, this further obscures any understanding of the bias. These should at least be listed in Table 2 or 3. 3. Within the NDACC, these types of retrieval parameters are clearly defined yet many stations apparently do no use them (Table 2) this seems counter productive to the goals of the network. 4. Also if the re- 
trievals are not performed to the NDACC standard are they indeed NDACC data? Are these data (meaning the 10y dataset from each station) found on the NDACC Archive? Or are they a separate retrieval? If they are not on the archive then they are not NDACC data and the premise of the manuscript is not at least completely valid.

The process to attain the anomaly plot Fig 5 is not described in sufficient detail. In particular the choice/method of terms in the annual cycles varying by site should be presented.

Pg 13 top paragraph. 'No systematic bias' except of course, for possible biases removed earlier. This 3 station biases mentioned (Tsukuba, Lauder and Arrival Heights) - How are these determined?

Figure 5 is difficult to determine a comparison. Correlation plots would better illustrate the good agreement and bias of the two datasets. These should be replaced.

\section{Minor issues}

There were many issues with the model that could be explored. For instance, the seasonal amplitude in $\mathrm{AH}$, Eureka \& Kiruna. The inability to reproduce the variability at Toronto. There was mention of, but no explanation for the annual cycle phase difference at Izana \& Tsukuba. There is a discussion of annual cycles and some mismatches (eg. JFJ, Izana). These are discussed only qualitatively when the data are available to quantify them. This would be an added benefit for the paper to put this in a table.

Its pointed out that the calculation of the trend at Wollongong could be effected by the early data variability. Could this early data not be removed from the trend calculation and compared as a shorter time series?

Pg 1 Ln 24 it appears misleading to state 'all members of the ...' The stations are all NDACC stations but not all NDAC station are used.

Pg 1 Ln 36 'secondary contributors' is poorly defined simply minor might be a choice. 
Pg1 Ln 41 its not clear what the reference for 0.97 is.

Pg 2 Ln 9 - The statement 'significant uncertainties'. Is this in a statistical sense? ACPD Can these uncertainties actually be stated in the text especially given the discussion of attribution later in the text. Are they known?

Pg 2 Ln 17 What is meant by "global surface climate change"?

Interactive

comment

$\mathrm{Pg} 3 \mathrm{Ln} 25$ Kiruna is not likely the most northern town in Sweden

Pg 4 - It may not be accurate to describe Toronto as a mega city.

Grammar, Spelling, Typographical issues

Pg 8 Ln 17 Fig 5.

$\mathrm{Pg} 17$ Ln 30 shown

Pg 18 Ln 13 sources not tracers.

Interactive comment on Atmos. Chem. Phys. Discuss., doi:10.5194/acp-2016-699, 2016. 